\title{
Electrical Imaging for Geohazard and Environmental Monitoring
}

\author{
Vincenzo Lapenna, ${ }^{1}$ Sabatino Piscitelli, ${ }^{1}$ and Pantelis Soupios ${ }^{2}$ \\ ${ }^{1}$ Institute of Methodologies for Environmental Analysis, National Research Council of Italy, 85050 Tito (PZ), Italy \\ ${ }^{2}$ Department of Natural Resources and Environment, Technological Educational Institute of Crete (TEI), Crete, Greece
}

Correspondence should be addressed to Vincenzo Lapenna, lapenna@imaa.cnr.it

Received 13 February 2012; Accepted 13 February 2012

Copyright ( 2012 Vincenzo Lapenna et al. This is an open access article distributed under the Creative Commons Attribution License, which permits unrestricted use, distribution, and reproduction in any medium, provided the original work is properly cited.

This special issue represents a contribute to disseminate the results of novel applications of the electrical resistivity imaging. Recently, innovative algorithms for tomographic data inversion and modern technologies for the field surveying have rapidly transformed the electrical imaging in a noninvasive, cost-effective, and powerful tool for geohazard and environmental monitoring. To date, the scientific interest for the electrical imaging is constantly growing and the novel approaches based on 3D and time-lapse 4D tomographic methods are disclosing the way for new and interesting applications, as well as the time-continuous mapping and monitoring of water content in a landslide body and the monitoring of weakly resistivity changes related to fluid migration processes close to seismic faults.

The papers published in this special issue mainly concern the study of hydrogeological problems typical of Mediterranean countries (i.e., landslide phenomena, saltwater intrusion) and the design of experiments for better evaluate the spatial and time-dependent changes of subsurface resistivity. Of course, these topics are not completely representative of all application fields of the electrical imaging.

In the paper of the special issue titled "Geoelectrical tomography investigating and modeling of fractures network around Bittit spring (Middle Atlas, Morocco)," Qarqori and other coauthors describe the main results of a geoelectrical survey carried out for monitoring a strategic aquifer in the northern part of Morocco. The study gives a significant contribute for defining the water flow in karst environment and through subvertical fractures.

The paper by T. Zhu et al., titled "Experimental studies on the changes in resistivity and its anisotropy using electrical resistivity tomography," deals with laboratory estimation on electrical anisotropy using electrical resistivity tomography on dry magnetite samples under uniaxial pressure.

The paper by A. Satriani et al., "Geoelectrical surveys for characterisation of the coastal salt water intrusion in Metapontum Forest Reserve (Southern Italy)," presents the results of a case study using resistivity imaging integrated with water and soil analysis to investigate salt water intrusion in a coastal aquifer, identifying large areas affected by intensive soil salinization, and delineating the saltwater intrusion front.

In the paper "Geoelectrical tomography as an operative tool for emergency management of landslide: an application in Basilicata Region, Italy," G. Colangelo and A. Perrone describe an electrical imaging survey, integrated with borehole data, to study a complex rototranslational slide, showing how the application of this indirect technique could be particularly useful for end users involved in the landslide risk management.

Finally, the paper "A prototype system for time-lapse electrical resistivity tomographies" by Luongo et al. concerns the first experimental results obtained with a new system for time-lapse resistivity monitoring of a landslide. The system seems to be suitable for water content monitoring in shallow layers.

Vincenzo Lapenna Sabatino Piscitelli Pantelis Soupios 

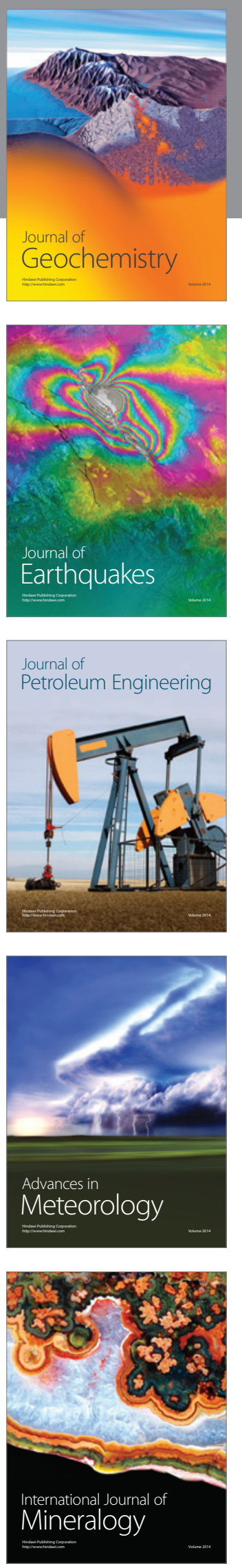
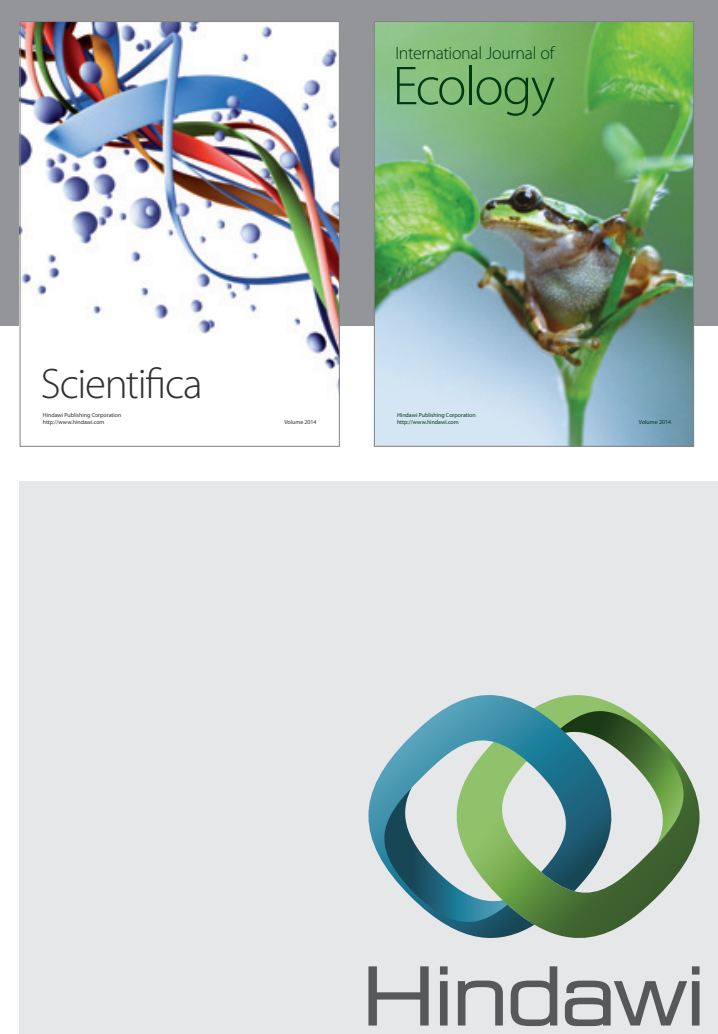

Submit your manuscripts at http://www.hindawi.com
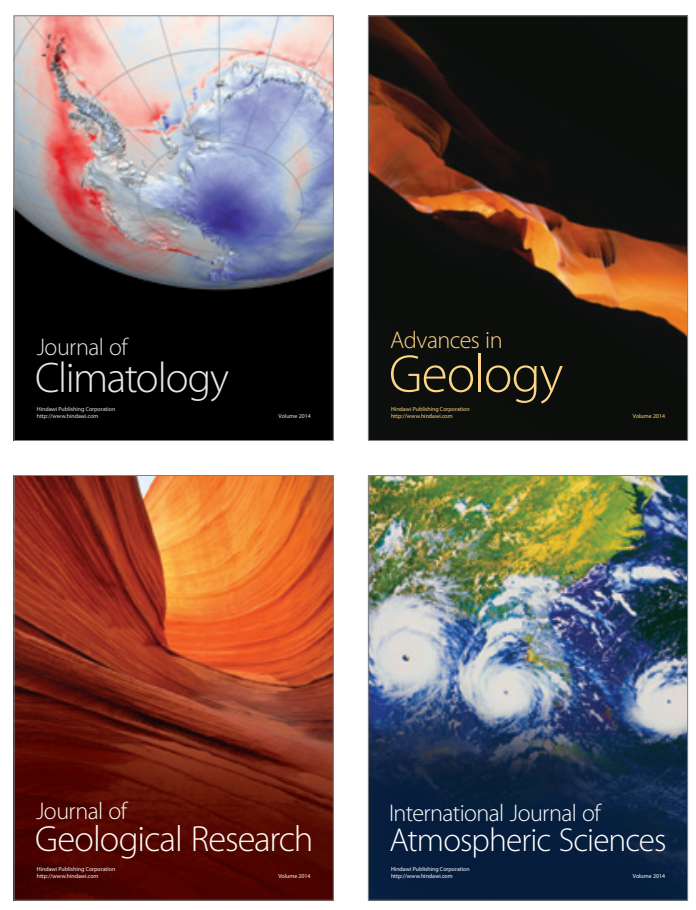
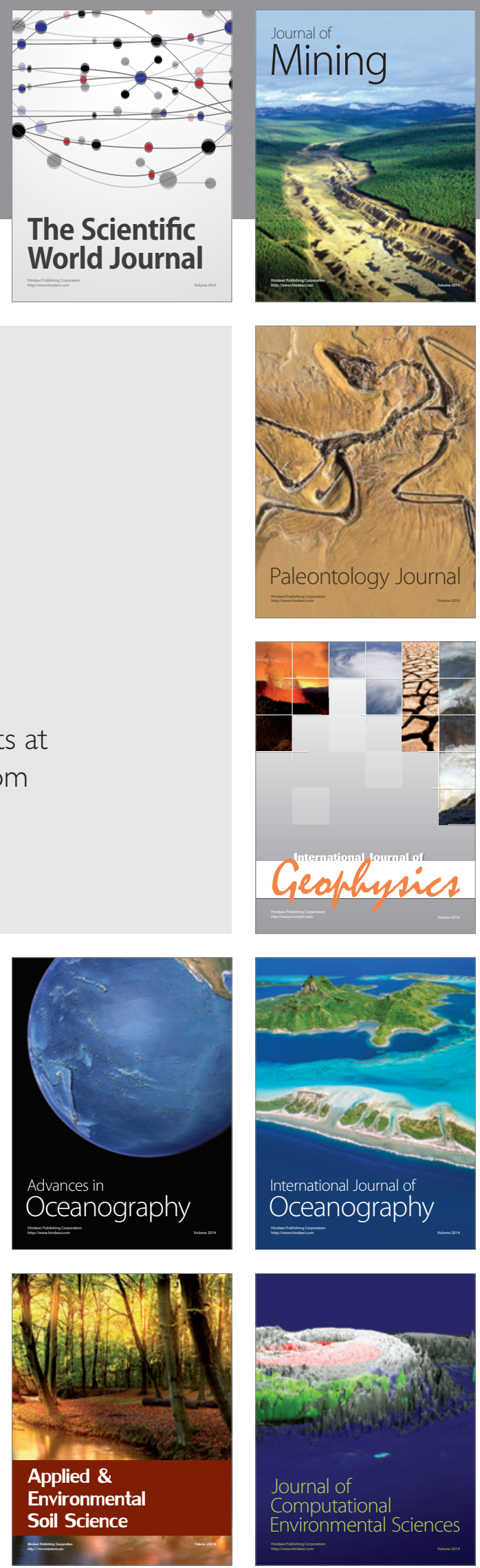